\title{
An improved wavelet correction for zero shifted accelerometer data
}

\author{
Timothy S. Edwards \\ Naval Surface Warfare Center, Dahlgren Division, Weapons Dynamics Branch, 17320 Dahlgren Road, Bldg. 489, \\ Rm. 110, Dahlgren VA, USA \\ Tel.: +1 540653 7347; E-mail: edwardsts@nswc.navy.mil
}

Received 2 January 2002

Revised 24 September 2002

\begin{abstract}
Accelerometer data from shock measurements often contains a spurious DC drifting phenomenon known as zero shifting. This erroneous signal can be caused by a variety of sources. The most conservative approach when dealing with such data is to discard it and collect a different set with steps taken to prevent the zero shifting. This approach is rarely practical, however. The test article may have been destroyed or it may be impossible or prohibitively costly to recreate the test. A method has been proposed by which wavelets may be used to correct the acceleration data. By comparing the corrected accelerometer data to an independent measurement of the acceleration from a laser vibrometer this paper shows that the corrected data, in the cases presented, accurately represents the shock. A method is presented by which the analyst may accurately choose the wavelet correction parameters. The comparisons are made in the time and frequency domains, as well as with the shock response spectrum.
\end{abstract}

\section{Introduction}

As documented many times before [1-4], the most conservative approach when dealing with zero shifted accelerometer data is to discard the corrupted data and re-acquire the data with precautions taken to prevent zero shifting. If the data are unique, however, it may not be possible to collect another set. Also, if measurement of small displacements is desired through integrating the accelerations, it becomes painfully obvious that many records obtained from accelerometers contain zero shifting to some degree. Under these circumstances it may become necessary to apply some correction technique to the accelerometer data to remove the spurious DC drifting.

Smallwood and Cap $[3,4]$ have presented several methods for the correction of accelerometer data including parametric filtering and wavelet-based corrections. With no independent measurement with which to compare the corrected data, the accuracy of the corrected data is judged by the plausibility of its integrations and Shock Response Spectrum (SRS). The cor- rected data in $[3,4]$ are shown to approach the accepted values for low frequency SRS and approach the appropriate final values of velocity and displacement. In doing so, the corrected accelerations are deemed credible, not necessarily correct.

This paper presents acceleration data corrected by the wavelet methods of Smallwood and Cap compared to non-contact measurements performed using a laser Doppler vibrometer (LDV). In this way, the corrected data may be evaluated on its accuracy, rather than its plausibility. This is the first known instance where zeroshifted accelerometer data has been evaluated by an independent measurement on its ability to be salvaged. A new method for choosing the severity of the wavedbased correction is also presented.

\section{Wavelet properties}

For a complete treatment of the wavelet transform the reader is directed to [5]. Other references on wavelets include [8], and [9]. Because the subject matter of 
this paper is experimentally collected data, and it is assumed that the actual signal has been sampled periodically, only the discrete wavelet transform (DWT) will be discussed. Like any other transformation the wavelet transform is accomplished by projecting the signal against the basis functions in the new space. In our case, the wavelet space is described by a single wavelet (sometimes called the mother wavelet) that undergoes dilations and translations. Each time the wavelet is dilated its support shrinks by a factor of two. For a given dilation, the wavelet then moves across the function in steps. At each step the projection is taken to determine the wavelet coefficient, $b_{j, k}$, where $j$ is the dilation parameter and $k$ is the translation parameter. As $j$ is incremented the wavelet's support shrinks by half. As $k$ is incremented the wavelet moves across the signal in time. If the correlation between the signal and the wavelet at a given dilation and translation is strong, the coefficient will be large. If the resemblance between the signal and wavelet is weak, the coefficient will be small. This operation is described by Eq. 1 .

$$
b_{j, k}=\left\langle x, w\left(2^{j} t-k\right)\right\rangle
$$

One efficient way to accomplish the DWT is by passing the sampled values through a logarithmic filter bank [5]. This process is illustrated in Fig. 1. The sampled signal, $x_{n}$, passes through one stage of the bank which consists of a high pass and low pass filter. The high pass filter contains terms that represent the wavelet shape function while the low pass filter represents the scaling function. The output of the high pass filter is known as the detail while the result of the low pass filtering operation is known as the approximation. After the first filter stage, the wavelet coefficients represent the finest details of the signal. If we wish to resolve the signal further, the approximation coefficients are fed into the next stage of the filter bank for another level of decomposition. The wavelet coefficients from this stage then represent signal components that are twice as large as the first. Therefore, this implementation of the DWT is recursive. This is possible because the space spanned by the scaling function at a given scale is also spanned by the wavelets at lower scales. If the signal has $2^{n}$ values, then there are usually n levels of decomposition available using the DWT. Because the output of each filter is of the same length as the original signal it is customary to downsample the signal by a factor of two after each filter. This results in a coefficients vector that is the same length as the original signal.

Once the wavelet decomposition has been made, we are free to alter the coefficients $b_{j, k}$ before performing the inverse transform. If we want to suppress the high frequency components of the signal, we can reduce the magnitude of the coefficients representing the finest details. We can even make them equal to zero if we like. Once the inverse transform is done, those portions of the signal represented by the altered coefficients will accordingly be reduced in magnitude. When the high frequency portions of the signal are suppressed this procedure is known as denoising.

If we desire to suppress low frequency components of the signal, we can likewise alter the coefficients representing them. This is exactly the method proposed by Smallwood and Cap [4] for correcting accelerometer zero shift. Since the acceleration records contain spurious low frequency drifting, we can simply eliminate the coefficients that represent large-scale portions of the signal. The high frequency portions, that hopefully represent actual accelerations, remain after the inverse transformation.

\section{Experimental}

An experiment was undertaken to produce zero shifted accelerometer records to investigate the accuracy of correction. The test fixture appears in Fig. 2. An aluminum plate was suspended between two steel blocks and fastened to the block at one end. The other end of the plate was allowed to vibrate against the steel block. An accelerometer was attached to the plate on one side while measurements were taken simultaneously on the opposite side using a laser Doppler vibrometer. The laser beam was aimed at the point directly beneath the accelerometer so that the two sensors would be measuring the same event within practical means. A steel ball bearing was then dropped on the aluminum plate. The height from which the bearing fell and the proximity of the impact location to the accelerometer were varied to produce zero shifts of different magnitudes. The fixture produced wide-band frequency content accelerations, similar to pyroshock. Several types of accelerometers were used including piezoelectric, piezoelectric with internal charge converter electronics, and piezoresistive. The data was sampled at 200,000 samples per second. The numerical differentiation was carried out using a finite impulse response (FIR) differentiating low-pass filter with a $20 \mathrm{kHz}$. cutoff frequency. Integration was done using the trapezoidal rule. 


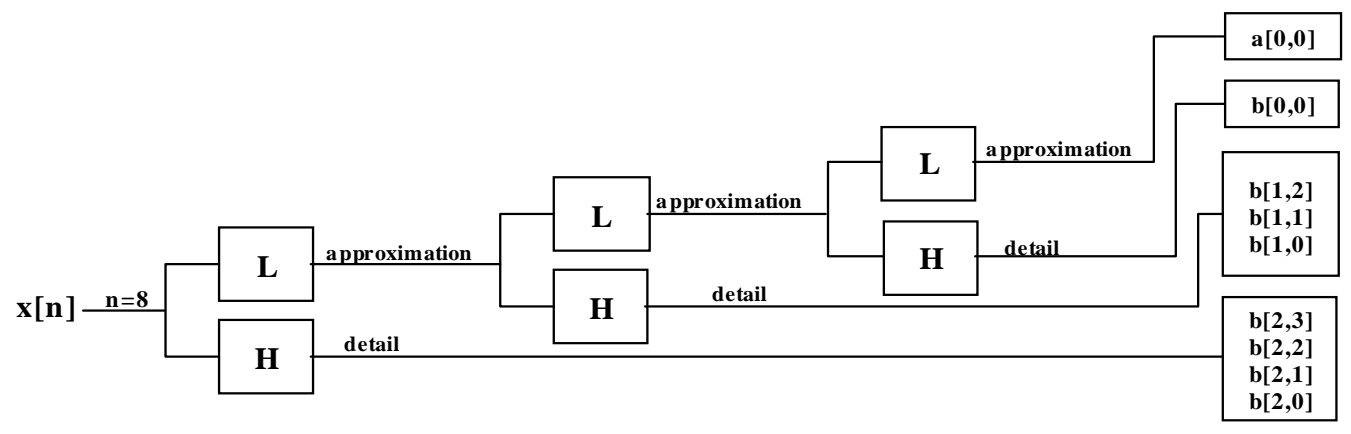

Fig. 1. Forward DWT operation.

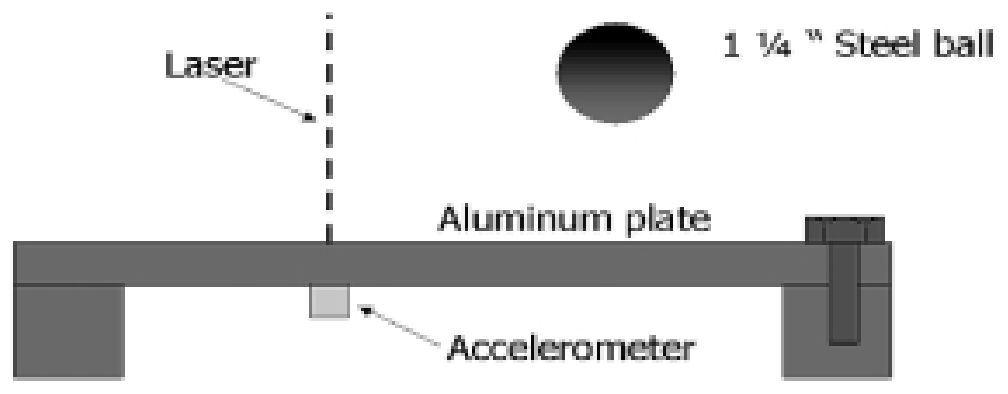

Fig. 2. Experimental setup.

\section{Traditional method for choosing correction parameters}

As with most techniques used to correct zero shifted accelerometer records, a common practice is to apply the correction in increasing severity to the acceleration data until the integrations yield physically plausible values. This is the method used in [4]. The acceleration data is transformed to the wavelet domain. The level of coefficients representing the largest scale of the signal are converted to zero and the inverse transform is computed. The data is then integrated to check the final values of velocity and displacement. If the integrations are still in error then the next level of wavelet coefficients representing the second largest-scale components of the signal are converted to zero. The inverse transform is performed again in order to check the integration. The method is repeated until the velocity and displacement achieve plausible values. The following example shows how use of this method can remove too much of the real acceleration data in addition to the zero shift.

Figure 3 shows a zero shifted accelerometer record, labeled Data Set 1, and its integrations. In the actual test, the accelerometer had no net displacement. Yet, the integration shows a false displacement of almost 50 millimeters. Also, the shock response spectrum, computed with 5\% critical damping, shows too much low frequency content given the source of the data. These are indications that a zero shift has occurred.

The acceleration data was decomposed into its wavelet coefficients and the coefficients representing the largest scale (low frequency) portions of the signal were forced to zero. The inverse transform was computed and the integrations performed. This process was repeated until the displacement record showed the correct final value - zero net displacement during the test. The displacement did not obtain this value until the signal was reconstructed using only the 5 highest of the 12 available levels. This is referred to in [4] as a level 5 correction. The resulting data is shown in Fig. 4. Now, the integrations and SRS take on plausible values.

Using only the integrations and low frequency range of the SRS as a guide we have seemingly done an acceptable job of correcting the data set. The acceleration data even looks about the same and the peak value of the corrected data is $99.7 \%$ that of the original data. Figure 5 shows the level 5 corrected data compared to the laser vibrometer in both displacement and the frequency domain. The displacements obtained from integrating the level 5 corrected accelerometer data are much smaller than the actual displacements. The Fourier spectrum shows the same trend. The cor- 

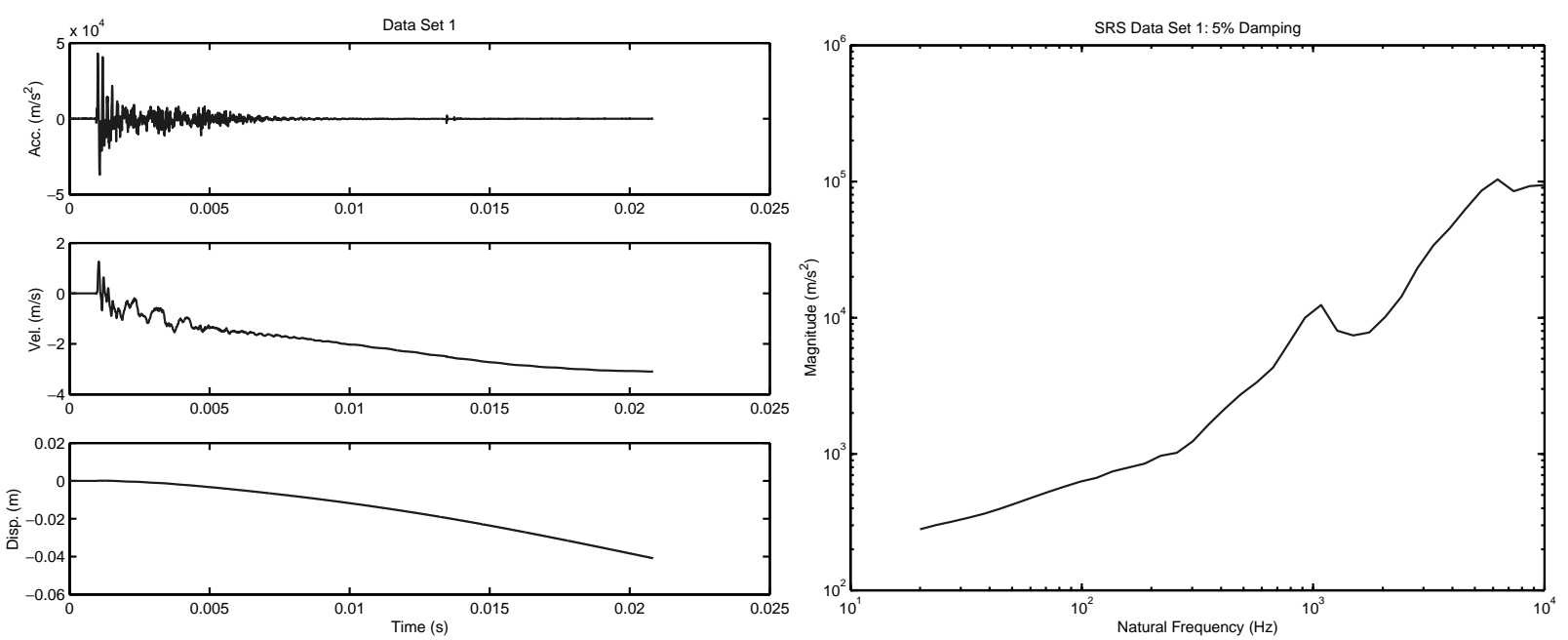

Fig. 3. Data Set 1.
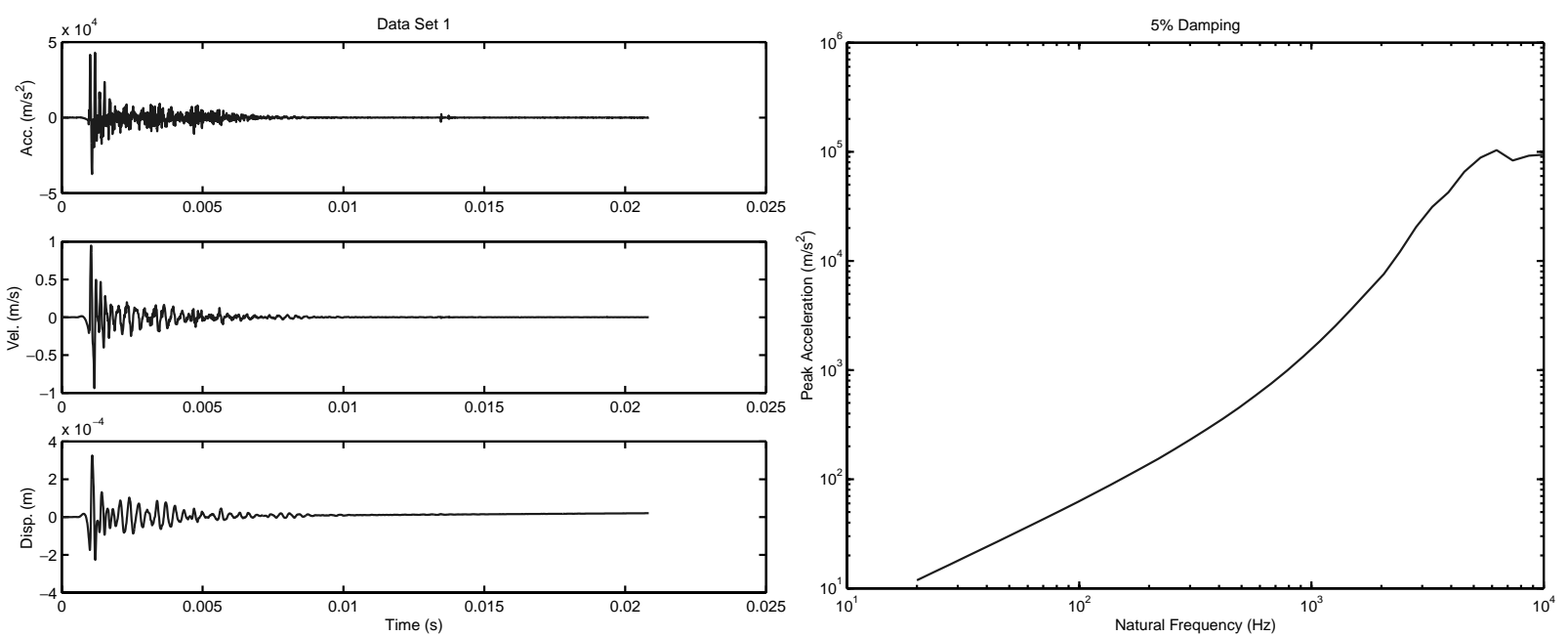

Fig. 4. Data Set 1, after Level 5 correction.

rected accelerometer data falls well below the spectrum of the acceleration as measured by the laser below $2 \mathrm{kHz}$. The laser spectrum falls off above $20 \mathrm{kHz}$, the cutoff frequency of the low pass, differentiating filter used to convert the laser velocity into acceleration data. From these charts, it is clear that the level 5 correction has removed too much real information from the accelerometer record.

Using the final values of velocity and displacement computed from the corrected accelerometer data to determine which wavelet levels to remove from the signal resulted in removing too much of the real acceleration in addition to the false low frequencies. But the excellent match between the Fourier spectrum of the zero shifted accelerometer data and the laser data above about $1 \mathrm{kHz}$ gives us hope that the actual accelerations might still be recovered from the zero shifted data. To develop a better approach for determining the wavelet correction level we turn to a few properties of the wavelets themselves.

\section{New method for choosing wavelet correction parameters}

To improve the correction scheme, we first invoke the property of vanishing moments. For a more complete treatment of vanishing moments, the reader is directed to [5] or [9]. Simply stated, a wavelet, $w(t)$, has $n+1$ vanishing moments if for $m=0, \ldots, n$ 

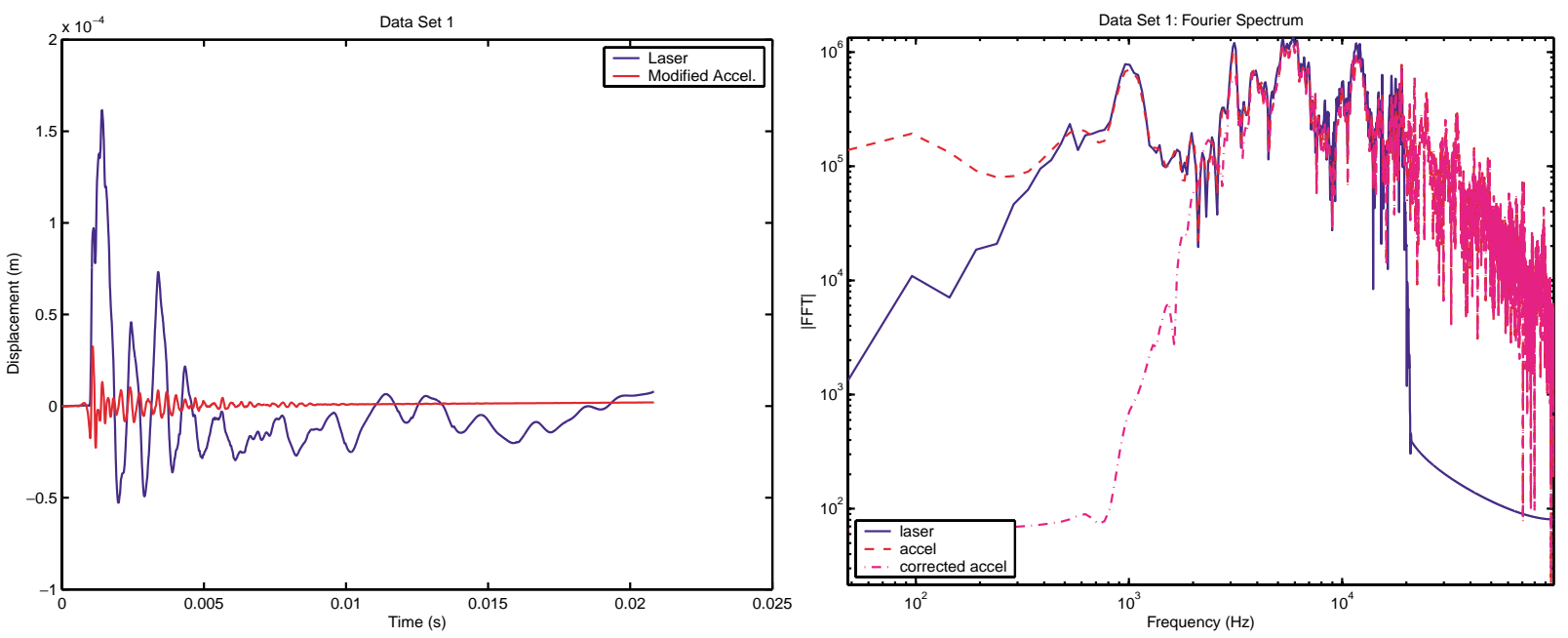

Fig. 5. Data Set 1, Level 5 correction and laser data.

$$
\int_{R} t^{m} w(t) d t=0
$$

All wavelets are required to have at least one vanishing moment, which means that they must poses zero net area. The number of vanishing moments a wavelet has generally increases with filter order. According to Eq. (2), the wavelet completely supresses any polynomial of order $n$ or less. A signal of polynomial order $n$ or less must be contained entirely in the space spanned by the scaling function (represented by the approximation coefficients). This comes about due to the orthogonality between the wavelet space and the space containing the scaling function.

Because we wish to represent (and subsequently remove) the zero shift as accurately as possible, and because we want to do so with the minimum number of coefficients as possible to avoid removing too much energy from the signal, we invoke a relation given by Strang in [5]. The relation gives a bound for the accuracy of the signal represented by the scaling functions at level $j$, for a wavelet with $n+1$ vanishing moments.

$$
\left\|f(t)-f_{j}(t)\right\| \leqslant C \cdot 2^{-j(n+1)}\left\|f^{(n+1)}(t)\right\|
$$

This relation says that the error between a function and its projection on the space spanned by the scaling functions at level $j$ is bounded by a constant, $C$, multiplied by the characteristic length of the level, $2^{-j}$, raised to the $n+1$ power, multiplied by the norm of the $n+1$ derivative of the signal. The derivative term tells us that the scaling functions will do a better job of representing the signal if the signal is smooth, and they will do it at a lower level of decomposition.
These two properties of wavelet analysis can lessen the probability that too much energy will be removed during the wavelet correction. The first property says that if we operate on a signal that looks like a polynomial, then, using a wavelet with sufficient vanishing moments we will be able to completely reject the polynomial components by forcing the approximation coefficients to zero and using only the detail coefficients in the inverse transform operation. The second property says that this suppression will be more successful, and we will have to force fewer coefficients to zero, if we operate on a smooth signal.

The heart of preserving the real signal energy while discarding the zero shift energy lies is operating on the integration of the signal rather than the signal itself. There are three primary reasons for this.

1. Integrating the function containing the zero shift makes the signal look very much like a polynomial, especially if we operate on the twice integrated record. We can suppress this polynomial.

2. The integration operation smooths the signal, making the approximation representation of the polynomial much more accurate. Smoothing also reduces the number of coefficients we will need to discard by making the approximation more accurate at lower levels.

3. Errors resulting from numeric integration are dependant on the normalized sample rate. The acceleration record, if not oversampled to a large degree, may still have unacceptable drift due to these integration errors [6,7]. Correcting the acceleration record and then integrating to find displacements requires that additional energies be 


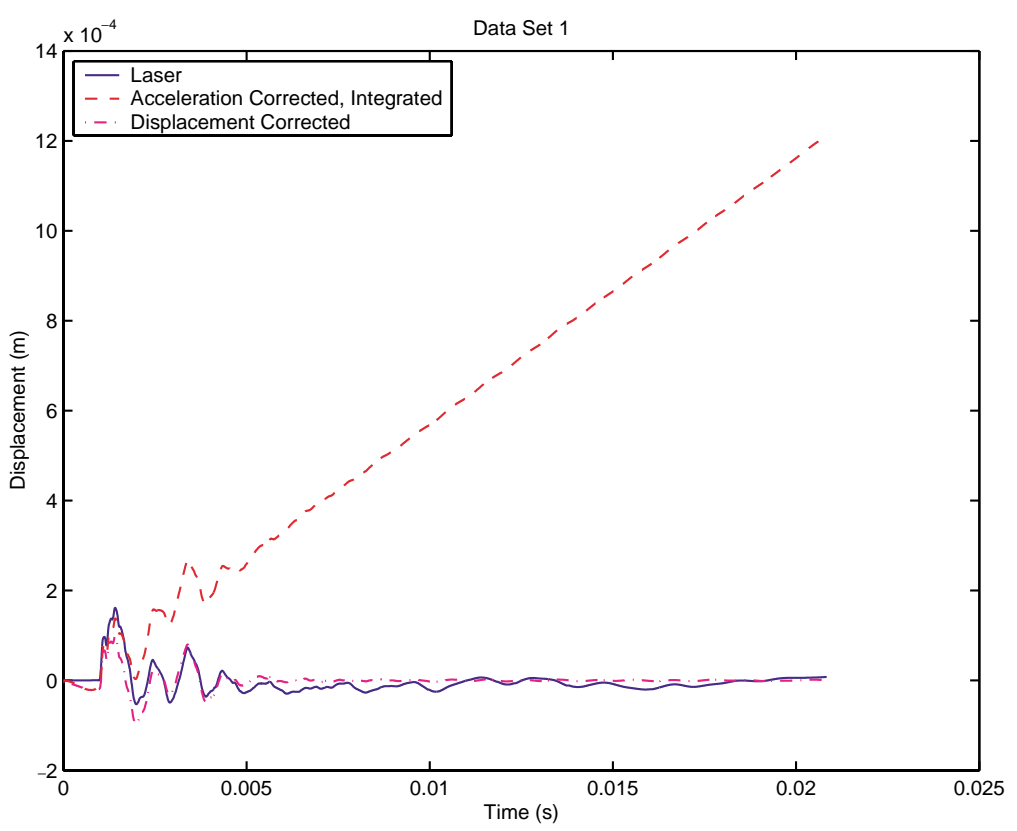

Fig. 6. Drift caused by integration error.

removed from the signal until the record ends at zero net displacement. But, since the drift is due to integration error, rather than a false low frequency that has been added to the signal, real signal components will be removed. So operating on the displacement record itself avoids the drift due to integration error and the false impression that a higher correction level is needed.

The proposed method for choosing the wavelet correction level simply involves correcting the displacement record, rather than operating on the acceleration record itself. The level of correction is still increased until the displacement achieves realistic values, but since we are using displacement, rather than acceleration, we are less likely to discard the parts of the signal we would like to preserve. Once the appropriate level of correction has been identified using the displacement record, the acceleration record is then corrected by decomposing it into the wavelet domain, forcing the previously identified coefficients to zero, and performing the signal reconstruction using the modified coefficients.

\section{Example}

The new procedure was used to correct the same data set shown in Fig. 3, the results are shown in Figs 6 and
7. Figure 6 shows the difference between operating on the acceleration versus operating on the displacement. When operating on the displacement, the level 8 correction was the first level of correction at which the displacements achieved zero net displacement at the end of the record. When a level 8 correction is performed on the acceleration record, and then the record is integrated to give displacement, Fig. 6 shows that the displacement still drifts undesirably. It should be noted that this performance is to be expected unless the acceleration record has been oversampled to a very high degree, and that this error is independent of the method of numerical integration used [6,7]. The false low frequency content added to the signal by the accelerometer zero shift has been removed by the correction procedure. This drift is due to numerical integration error.

If we were to choose the severity of the correction by operating on the acceleration record, one would naturally increase the level correction and subsequently remove real information from the data. As shown in Fig. 4, we must discard 3 more levels of coefficients when operating on the acceleration record before its integrated values are plausible. But, these three levels contain real information that should not be discarded.

Figure 7 shows the remaining comparisons made between the laser and the accelerometer. The bottom right chart shows the corrected acceleration data plotted with the laser velocity output, differentiated to yield acceleration. The two are so similar that they appear almost as 


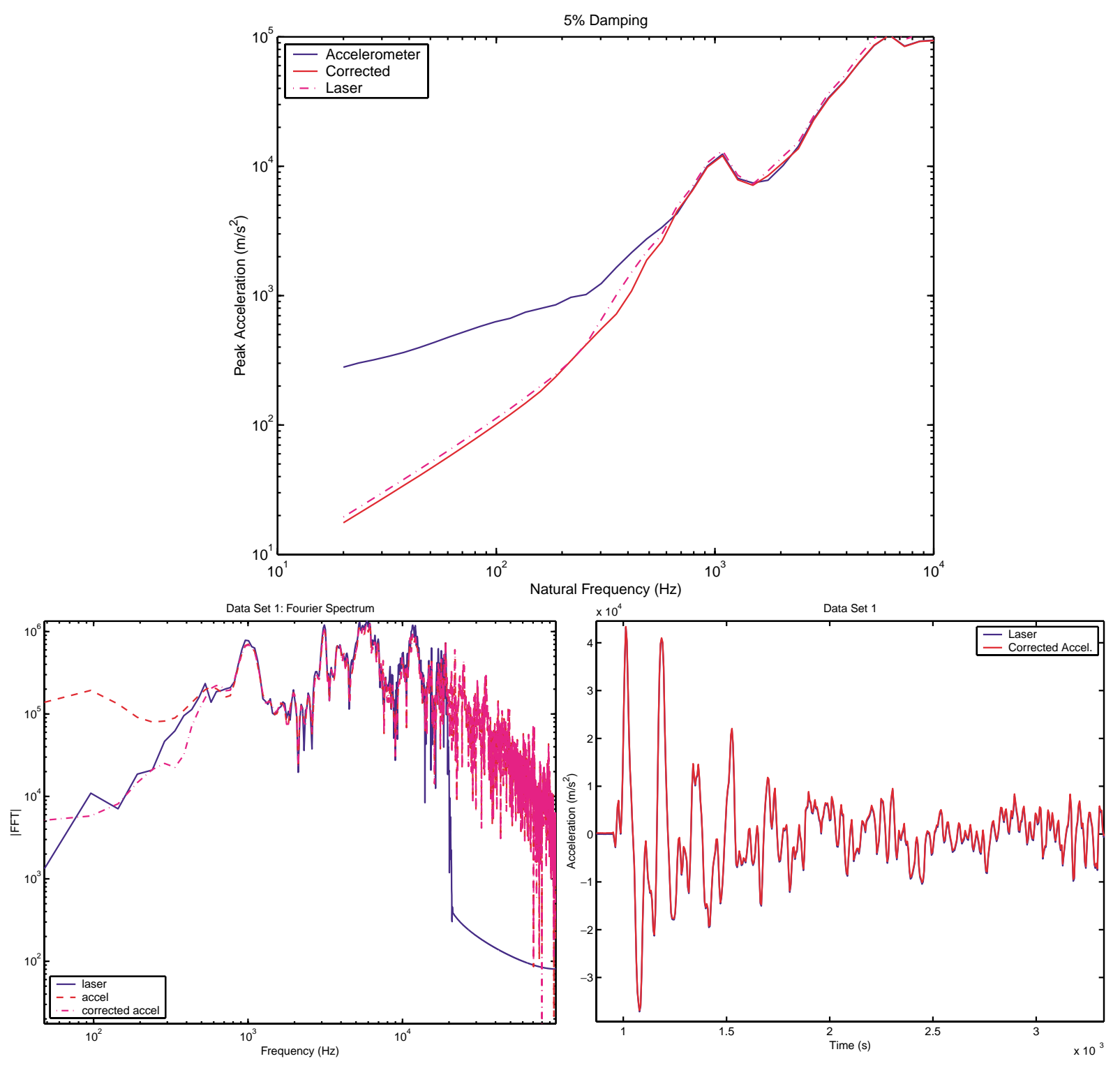

Fig. 7. Data Set 1, corrected using new method.

a single line on the plot. The chart on the bottom right is the Fourier spectrum of the original and corrected accelerometer data plotted with the differentiated laser velocity. Again, the spectrum of the differentiated laser velocity drops off at $20 \mathrm{kHz}$, the cutoff frequency of the low-pass, differentiating filter used to process the data. The top chart is the shock response spectrum of the original and corrected accelerometer data plotted with the shock response spectrum of the differentiated laser velocity. The corrected acceleration and the laser show very good agreement and have appropriate levels given the nature of the experiment. Similar results were achieved for all the data sets collected during this study.

The LDV used during this study gives independent outputs for displacement and velocity. The velocity output is derived from a Doppler phase shift measurement, while the displacement output is from a fringe counting technique. The two quantities are independent of each other and are not merely integrated or differentiated versions of the same measurement. Given this, a reasonable check on the accuracy of the measured laser data and the numerical routines used to process it to compare to the accelerometer data consists of 

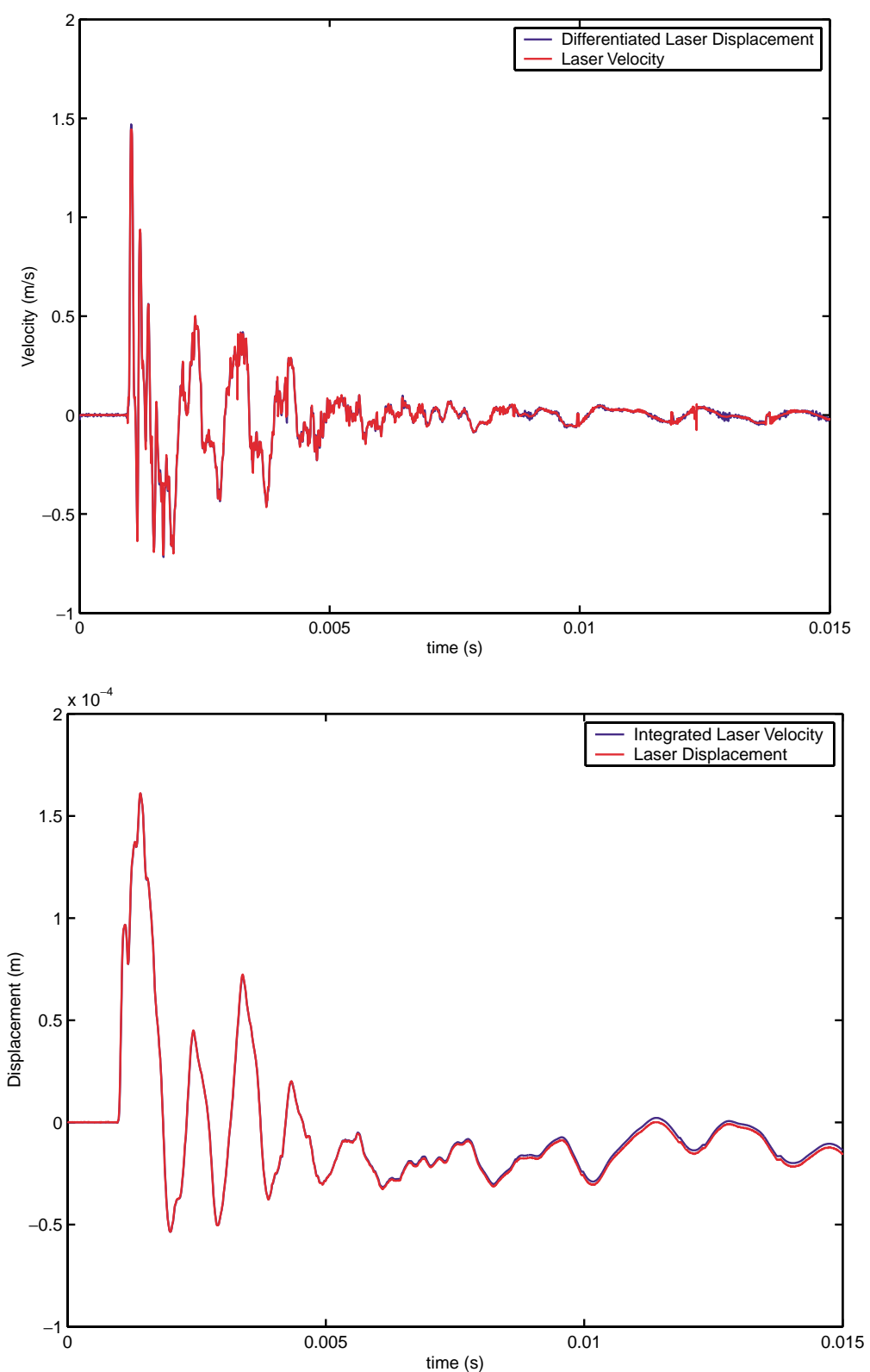

Fig. 8. Validation of LDV and integration and differentiation techniques.

differentiating the laser displacement and comparing to the velocity output, and integrating the velocity output for comparison with the displacement measurement. Figure 8 shows just such a comparison. The results are so similar that there is almost no way to distinguish them on the plots. The small differences at the end of the displacement record are attributable to visible noise spikes in the velocity record. These results were similar no matter what integration or differentiation method was used.

\section{Summary}

Using wavelets to correct accelerometer data is a powerful technique. Choosing the correction level is based on the displacement value taking on the value of the true displacement encountered during the test. For the data presented, and for the majority of pyroshock data, the test article has zero net displacement at the end of the test. This information can be used to gage the validity of the original and corrected data. When choos- 
ing the severity of the correction, and using SRS and integration as a measure, operating on the displacement is more accurate than if the acceleration is corrected and then integrated to check the correction level.

There has been debate over the validity of correcting zero shifted accelerometer data. Using an independent measurement, it has been shown that correcting accelerometer data may indeed be appropriate. It is acknowledged that many sources of zero shift exist as documented in [2] and that the data collected in this study is anecdotal evidence. There is no way to prove that every case of zero shifting may be corrected without impunity. However, for the zero shifting encountered in this study the data presented is typical. Despite the zero shift, the actual acceleration record could be recovered very accurately. In fact, the zero shifts were apparently wholly linear and additive in nature, and as such, could be removed to recover the true acceleration data.

While this paper presents an optimistic view of the correction of zero shifted accelerometer data, it is acknowledged that there are risks involved as documented by Galef [1]. No correction technique can account for information lost during the measurement. While the methods presented in this paper worked remarkably well for numerous data sets studied, the evidence is still anecdotal. There may be forms of zero shifting not induced by this experiment that are not correctable. With caveats being stated, this paper presents some of the first evidence that suggests the zero shifting phenomenon in its milder forms may be treated as a linearly additive effect.

\section{References}

[1] A. Galef, Zero-Shifted Accelerometer Outputs, 56th Shock and Vibration Symposium, pp. 77-79.

[2] A. Chu, Zeroshift of Piezoelectric Accelerometers in Pyroshock Measurements, 57th Shock and Vibration Symposium.

[3] D.O. Smallwood and J.S. Cap, Salvaging Transient Data with Overloads and Zero Offsets, 68th Shock and Vibration Symposium, 1998 SAVIAC, Arlington, VA.

[4] D.O. Smallwood and J.S. Cap, Salvaging Pyrotechnic Data with Minor Overloads and Offsets, Proceedings of the Institute of Environmental Sciences, 1998.

[5] G. Strang and T. Nguyen, Wavelets and Filter Banks, WellesleyCambridge Press, Wellesley, MA, 1997.

[6] T.S. Edwards and A.L. Wicks, Numerical Integration and Differentiation Techniques: A Comparative Study, submitted 21st International Modal Analysis Conference.

[7] D.O. Smallwood, Correcting Numerical Integration Errors Caused by Small Aliasing Errors, 68th Shock and Vibration Symposium, 1998 SAVIAC, Arlington, VA.

[8] I. Daubechies, The Wavelet Transform, Time Frequency Localization and Signal Analysis, IEEE Trans. Inf. Theory 36 (1990), 961-1005.

[9] S. Mallat, A Wavelet Tour of Signal Processing, Academic Press, New York, 1999. 

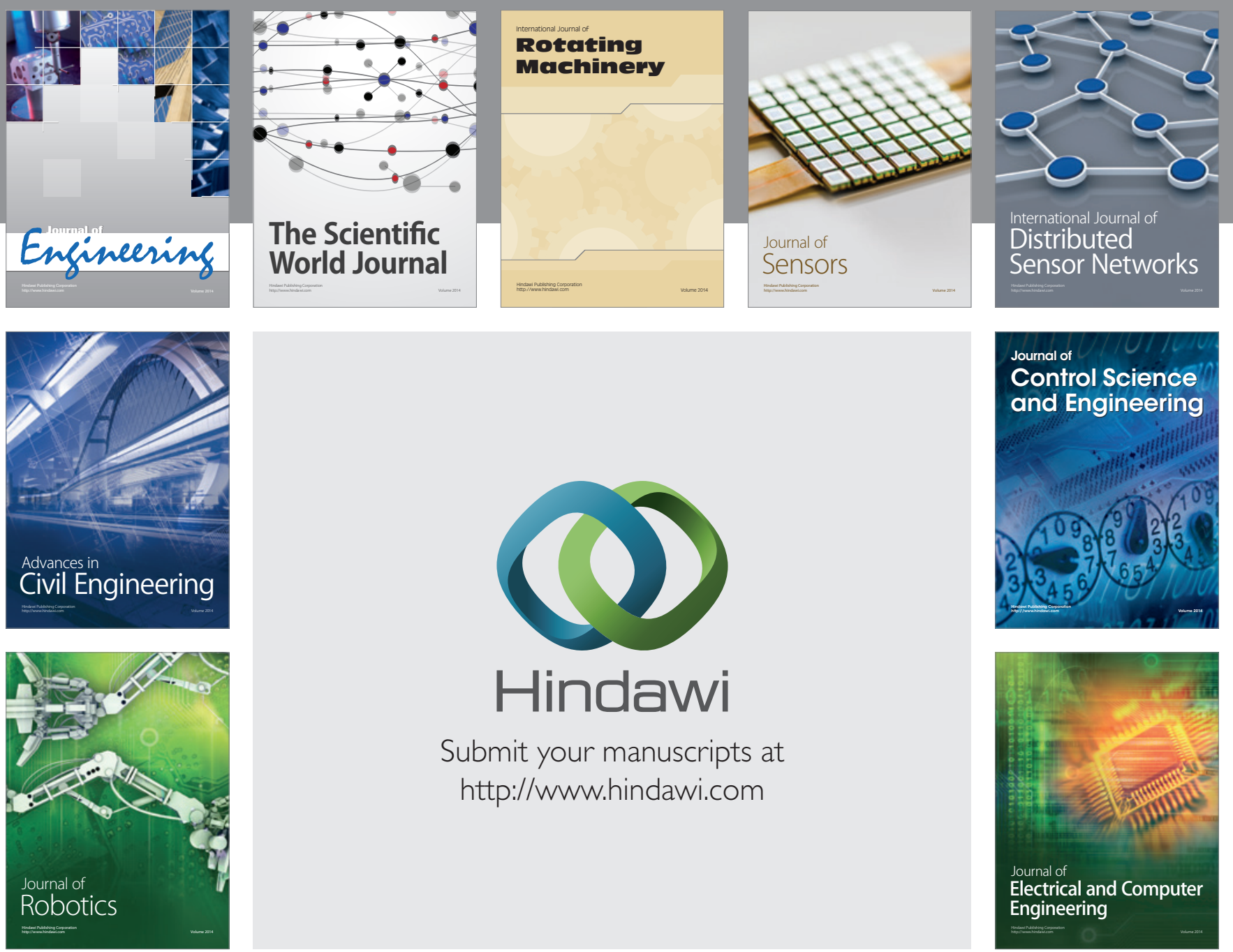

Submit your manuscripts at

http://www.hindawi.com
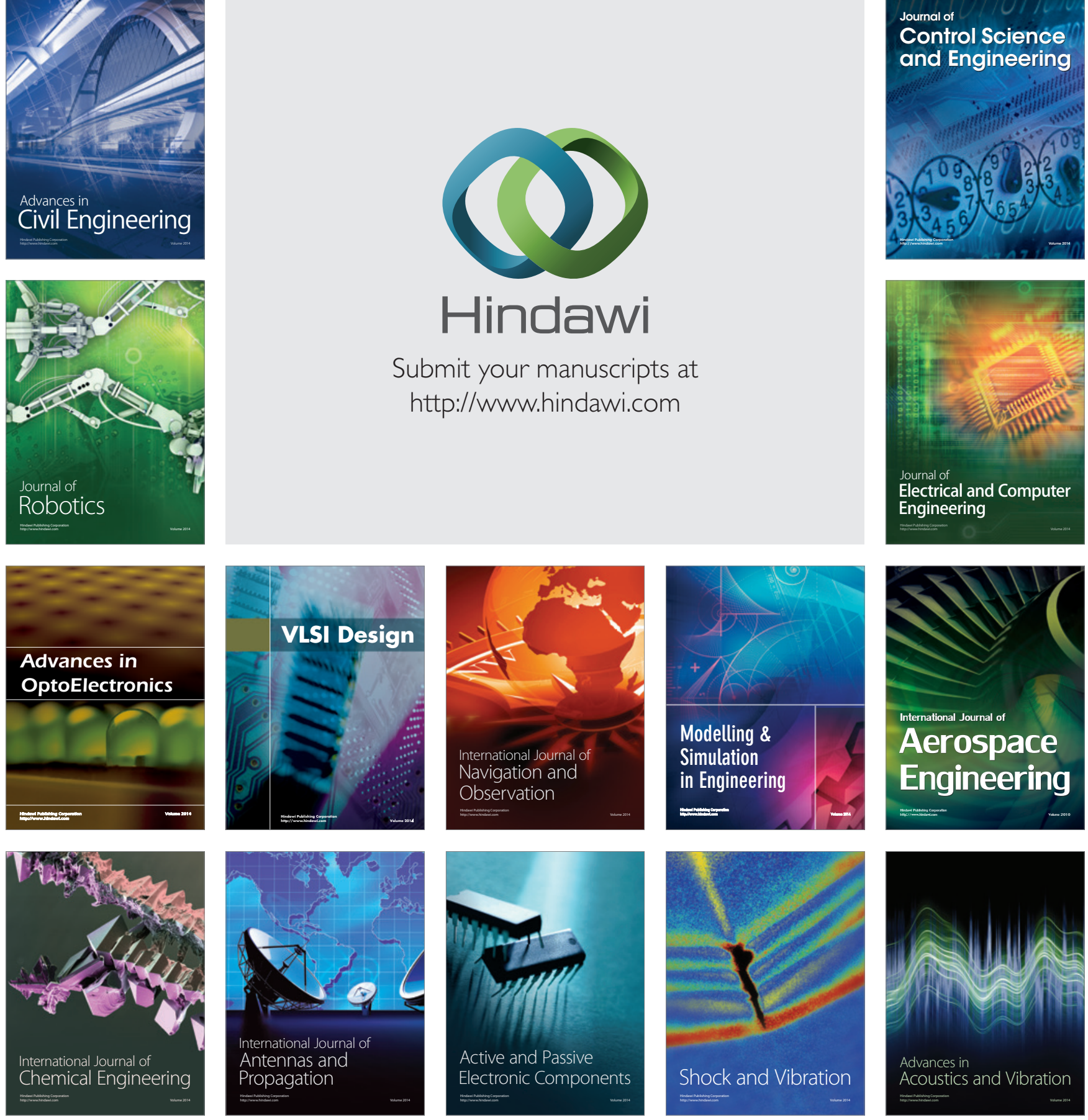